[white paper]

Diamond Open Access

\title{
Prova da inseparabilidade do emaranhamento máximo: um algoritmo de resolução
}

\author{
Colaboração Ciência Aberta ${ }^{1}$ \\ 27 de Setembro de 2021
}

\begin{abstract}
Resumo
Apresentamos um algoritmo de resolução, com o intuito de guiar - leitor na demonstração da inseparabilidade de duas partículas maximamente emaranhadas.
\end{abstract}

palavras-chave: emaranhamento máximo, bit quântico, qubit, teoria da informação quântica

A versão mais atualizada deste artigo está disponível em

https://osf.io/vcx8b/download

https://zenodo.org/record/5533018

\section{Preâmbulo}

1. Este white paper [1,2] é uma adaptação de [3], transposto na forma de um Algoritmo de Resolução, seguindo a licença [4].

2. Um Algoritmo de Resolução é um material de cunho didáticopedagógico, que está entre a resolução completa e o gabarito de um problema.

${ }^{1}$ Todos os autores com suas afiliações aparecem no final deste artigo. 


\section{Introdução}

3. Um bit quântico (qubit) é representado por $|\phi\rangle=\alpha|0\rangle+\beta|1\rangle$.

4. $\alpha$ e $\beta$ são números complexos, satisfazendo $|\alpha|^{2}+|\beta|^{2}=1$.

5. $|0\rangle$ e $|1\rangle$ são estados quânticos.

6. (5) pode caracterizar o spin (para cima/baixo), a polarização (horizontal/vertical), por exemplo.

7. Um sistema de duas partículas é descrito pelo produto tensorial,

$$
|0\rangle \otimes|1\rangle \equiv|0\rangle|1\rangle \equiv|01\rangle .
$$

8. Em (7), a partícula 1 está no estado $|0\rangle$, e a partícula 2 está no estado $|1\rangle$.

\section{Emaranhamento máximo}

9. O seguinte estado de Bell representa o emaranhamento máximo

$$
|\psi\rangle=\alpha|01\rangle+\beta|10\rangle,
$$

onde: $\alpha, \beta \in \mathbb{C} ; \alpha \neq 0, \beta \neq 0 ;|\alpha|^{2}+|\beta|^{2}=1$.

10. O sistema (9) é composto por duas partículas, cada uma pode ser medida em um dos dois estados, $|0\rangle$ ou $|1\rangle$.

\section{Inseparabilidade}

11. Suponha que $|\psi\rangle$ em (9) seja separável, i.e.,

$$
|\psi\rangle=|A\rangle|B\rangle .
$$

12. Considere que a partícula $A$ esteja na superposição quântica

$$
|A\rangle=a|0\rangle+b|1\rangle .
$$


13. E considere que a partícula $B$ esteja no estado

$$
|B\rangle=c|0\rangle+d|1\rangle .
$$

14. $a, b, c, d \in \mathbb{C}$ são as amplitudes para os estados quânticos $|A\rangle$ e $|B\rangle$, $\operatorname{com}|a|^{2}+|b|^{2}=1$, e $|c|^{2}+|d|^{2}=1$.

15. As seguintes condições são obrigatórias para que $A$ e $B$ estejam em uma superposição na base computacional $|0\rangle$ e $|1\rangle$,

$$
a \neq 0, b \neq 0, c \neq 0, d \neq 0 .
$$

16. Explique, a seguir, o porquê da condição (15). 
17. Insira (12) e (13) em (11), e mostre que

$$
|\psi\rangle=a c|00\rangle+a d|01\rangle+b c|10\rangle+b d|11\rangle ;
$$

inclua todas as passagens matemáticas. 
18. Iguale (9) a (17) e obtenha

$$
\alpha|01\rangle+\beta|10\rangle=a c|00\rangle+a d|01\rangle+b c|10\rangle+b d|11\rangle ;
$$

qual item justifica esse passo e por quê? 
19. Compare os termos que multipicam o estado $|01\rangle$ em (18) e mostre que $\alpha=a d$.

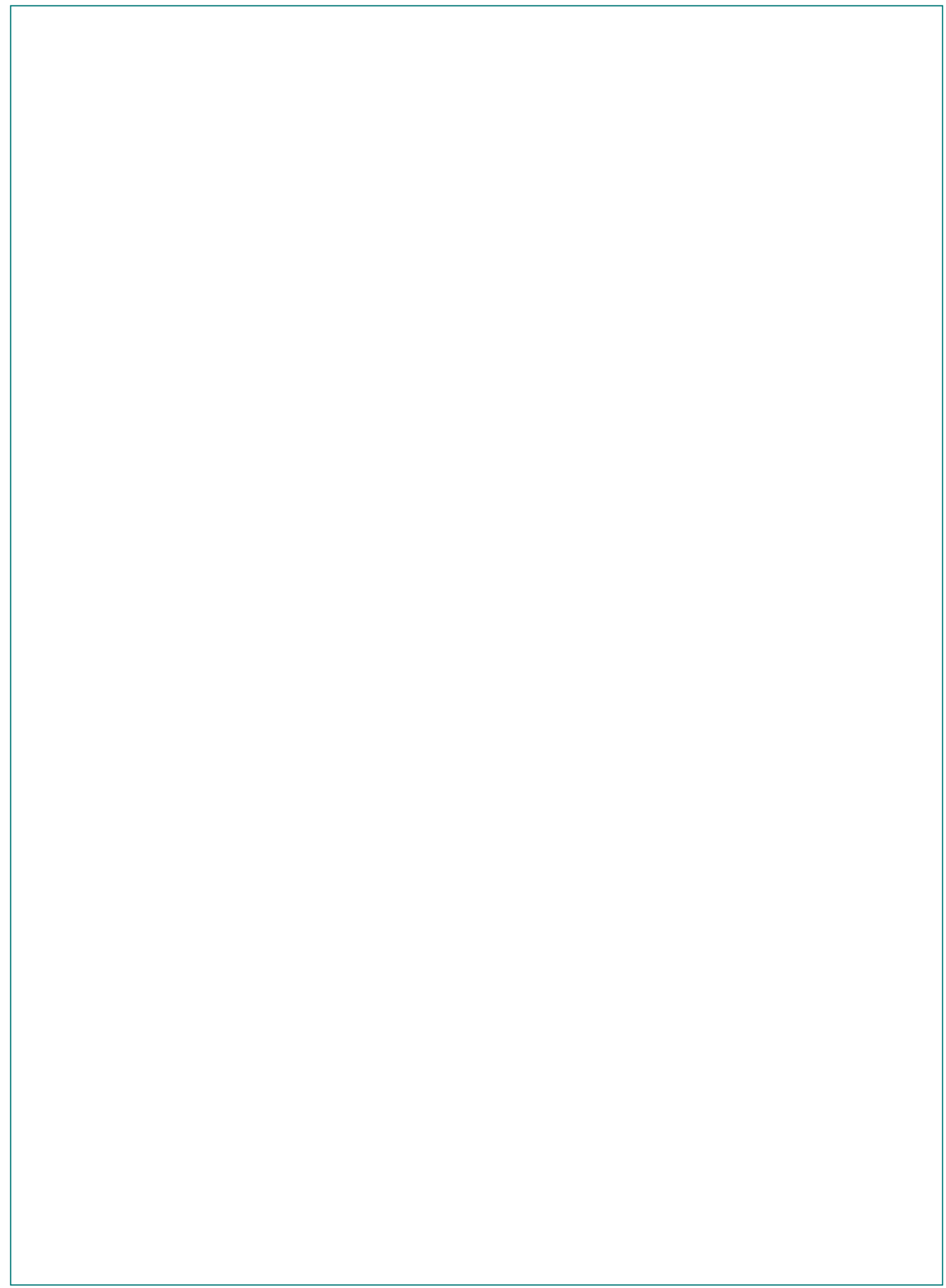


20. Faça o mesmo que em (19), mas para o estado $|10\rangle$, e mostre que $\beta=b c$.

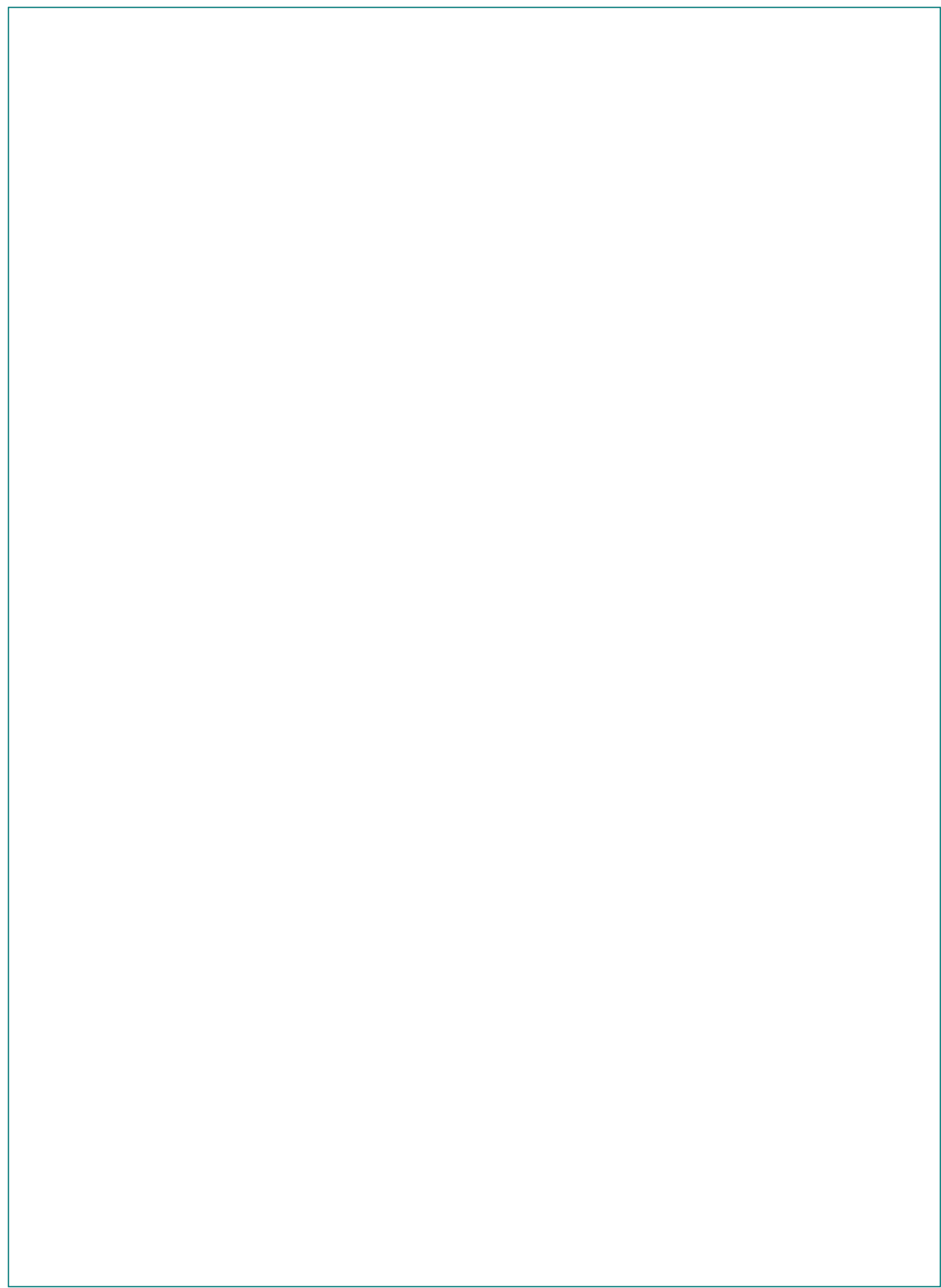


21. Analisando o lado esquerdo de (18), explique, considerando os estados $|00\rangle$ e $|11\rangle$, por que $a c=0$ e $b d=0$. 
22. Podemos dizer que o resultado obtido em (21) contradiz o resultado obtido em (15)? Justifique. 
23. Com base na resposta que você deu em (22), podemos dizer que a hipótese (11) está correta ou incorreta? Por quê? 
24. Considerando a resposta que você deu em (23), isso prova que $|\psi\rangle$ em (9) é um estado quântico separável, dado por

$$
|\psi\rangle=|A\rangle|B\rangle,
$$

ou inseparável, dado por

$$
|\psi\rangle \neq|A\rangle|B\rangle ?
$$


25. Na matemática, a demonstração finalizada em (24) é denominada prova por ...?

\section{Superposição de dois qubits}

26. Considere $\left|\psi_{2}\right\rangle$ na seguinte superposição,

$$
\left|\psi_{2}\right\rangle=m|00\rangle+n|01\rangle+p|10\rangle+q|11\rangle,
$$

onde $m, n, p, q \in \mathbb{C}$ e são todos diferentes de zero.

27. $\left|\psi_{2}\right\rangle$ é separável? Por quê? Se sim, escreva-o em função dos estados separáveis. 


\section{Considerações Finais}

28. O emaranhamento quântico é um dos recursos mais importantes da teoria da informação quântica [5-7].

29. O próprio espaço-tempo parece estar entrelaçado [8].

30. Na teoria da relatividade especial, sabemos que espaço e tempo são vinculados por $\tau^{2}=t^{2}-x^{2}[9,10]$, onde $t$ e $x$ são a duração e a separação espacial, respectivamente, entre dois eventos quaisquer.

31. Além disso, considerando o princípio da incerteza, espaço e matéria estão emaranhados, bem como energia e tempo.

\section{Ciência Aberta}

O arquivo latex para este artigo, juntamente com outros arquivos suplementares, estão disponíveis em [11]. Seja coautor(a) deste artigo, envie sua contribuição para mplobo@uft. edu.br.

\section{Consentimento}

Os autores concordam com [12].

\section{Como citar este artigo?}

https://doi.org/10.31219/osf .io/vcx8b

https://zenodo.org/record/5533018

\section{Licença}

CC-By Attribution 4.0 International [4] 


\section{Referências}

[1] Lobo, Matheus P. "Uma Revista Aberta." OSF Preprints, 19 July 2021. https://doi.org/10.31219/osf.io/fjb9a

[2] Lobo, Matheus P. "Microarticles." OSF Preprints, 28 Oct. 2019. https://doi.org/10.31219/osf.io/ejrct

[3] Lobo, Matheus P. "Proof of the Inseparability of Maximal Entanglement." OSF Preprints, 20 July 2019. https://doi.org/10.31219/osf.io/aejm3

[4] CC. Creative Commons. CC-By Attribution 4.0 International. https://creativecommons.org/licenses/by/4.0

[5] Nielsen, Michael A., and Isaac Chuang. Quantum computation and quantum information. Cambridge University Press, 2010.

[6] Sutor, Robert S. Dancing with Qubits: How quantum computing works and how it can change the world. Packt Publishing Ltd, 2019.

[7] Bernhardt, Chris. Quantum computing for everyone. MIT Press, 2019.

[8] Van Raamsdonk, Mark. "Building up spacetime with quantum entanglement." General Relativity and Gravitation 42.10 (2010): 2323-2329.

[9] Lorentz, Hendrik Antoon, et al. The principle of relativity: a collection of original memoirs on the special and general theory of relativity. Courier Corporation, 1952.

[10] Taylor, Edwin F., Edwin F. Taylor, and John Archibald Wheeler. Spacetime Physics. Macmillan, 1992.

[11] Lobo, Matheus P. "Open Journal of Mathematics and Physics (OJMP)." OSF, 21 Apr. 2020.

https://doi.org/10.17605/osf.io/6hzyp 
[12] Lobo, Matheus P. "Simple Guidelines for Authors: Open Journal of Mathematics and Physics." OSF Preprints, 15 Nov. 2019. https://doi.org/10.31219/osf .io/fk836

\section{Colaboração Ciência Aberta}

Matheus Pereira Lobo (autor principal, mplobo@uft.edu.br) $)^{1,2}$ https://orcid.org/0000-0003-4554-1372

\section{Lídia Cruz de Araújo ${ }^{1}$}

${ }^{1}$ Universidade Federal do Tocantins (Brasil)

${ }^{2}$ Universidade Aberta (UAb, Portugal) 